\title{
"Minoría bulliciosa". Periodismo feminista en tiempos de precarización laboral y reacción patriarcal
}

"Bustling minority". Feminist journalism in the times of precariousness at job and patriarchal reaction

\author{
Florencia Laura Rovetto \\ Universidad Nacional de Rosario. Centro de Investigaciones de Investigaciones Feministas y Estudios de Género I \\ Universidad Nacional de Entre Ríos. Facultad de Trabajo Social - CONICET, Argentina \\ florencia.rovetto@gmail.com

\section{Lucia Anabi Figueroa} \\ Universidad Nacional de Rosario. Centro de Investigaciones de Investigaciones Feministas y Estudios de Género - \\ CONICET, Argentina \\ lulafigueroa87@gmail.com
}

\section{ReSUMEN:}

En esta comunicación, nos adentramos en la labor periodística tomando la experiencia de jóvenes mujeres profesionales que participan de la escena mediática "emergente" produciendo contenidos relacionados con la agenda de reivindicaciones de los movimientos de mujeres y de la diversidad, al mismo tiempo que interpelan las discriminaciones de género en los espacios tradicionales de producción informativa. En ese recorrido se evidencia que tales productoras de contenidos informativos -"periofeministas"-, realizan una tarea que es profesional y militante al mismo tiempo, en un contexto signado por el ajuste, la flexibilización y precarización laboral que afecta al sector. En acercamientos previos al campo, hemos podido caracterizar el trabajo de las perio-feministas como una articulación política indispensable en la disputa contrahegemónica cultural frente a los discursos y las prácticas androcéntricas dominantes en los medios de comunicación masiva tradicionales. Aquí, nos proponemos explorar el trabajo que realizan en espacios mediáticos emergentes, concebidos desde su origen como alternativos, comunitarios y/o cooperativos para analizar las particularidades de las rutinas productivas y las condiciones de trabajo, observando contrastes y continuidades con los espacios de producción informativa de medios hegemónico o tradicionales.

Palabras clave: Periodismo, Feminismo, Medios Alternativos de Comunicación, Argentina.

\section{ABSTRACT:}

In this communication we analyze female journalists' work by pondering the experience of professionals who participate in "emergent" media scene, putting on media agenda crucial issues linked to women's, trans', lesbians' and bisexuals' rights, at the same time that they the gender discriminations into their information-production workplaces. It's evident that these journalists -or "Femi-journalist"- perform a professional and activist labor, in a context signed by the the overload of working hours, precariousness at job and its impact at this laboral sector. In previous approaches to the field, we have been able to characterize the work of these feminists as an indispensable political articulation in the cultural battle against the androcentric speech and practices of traditional media. Here, we propose to explore feminist's journalist work at emerging media spaces (conceived as "alternative", self-managed or cooperative media), to analyze the particularities of working conditions of productions routines, observing contrasts and continuities with the characteristic of traditional media.

KeYwORDs: Journalism, Feminism, Alternative Media, Argentina. 


\section{INTRODUCCIÓN}

El interés por analizar cómo se manifiestan las desigualdades de género en la producción mediática en general, enfocando de forma particular los espacios laborales de producción periodística, tiene ya algunos años de desarrollo (Gallego, 2002; Ortega y Humanes, 2000; López Díez, 2004; Papí Gálvez, 2008; Soriano, 2005;) en otras latitudes, y cuenta con algunos desarrollos más reciente a nivel local (Amado, Bongiovanni y Dragonetti, 2016; Rovetto, 2013 y 2017; Rovetto y Figueroa, 2015), ${ }^{1}$ así como con diagnósticos elaborados por organizaciones extra académicas como el Proyecto de Monitoreo Global de Medios (GMMP, por sus siglas en inglés), que monitorea el sesgo de género en los contenidos periodísticos, analizando porcentaje de autorías y de fuentes consultadas en una jornada informativa completa, cada cinco años en más de 100 países, en todo el mundo.

En el último informe GMMP, realizado en 2015, se señala que, en la prensa gráfica, las reporteras argentinas alcanzan, en promedio, el 15\% de las notas firmadas. A pesar de la creciente incorporación de mujeres en la producción periodística, Argentina tiene el promedio de firmas más bajas de América Latina. Solo en Bolivia y México ostentan paridad. En el resto de los países latinoamericanos la estampa presenta distintos niveles de inequidad. En Chile, el porcentaje de mujeres reporteras que firman noticias duplica a la Argentina y en Paraguay, es del 25\% (WACC, 2015).

Por otra parte, en aproximaciones previas al campo, indagando aspectos vinculados a la incidencia de la sanción de dos leyes de carácter nacional: la Ley 26.485 de Protección Integral para Prevenir, Sancionar y Erradicar la Violencia contra las Mujeres en los Ámbitos en que Desarrollen sus Relaciones Interpersonales y la Ley 26.522 de Servicios de Comunicación Audiovisual -que en su plexo incluyen referencias al derecho comunicacional de las mujeres y de colectivos discriminados por razones de género- ${ }^{2}$ sostuvimos que ambos instrumentos legales ofrecían un marco propicio para promover la perspectiva de género en los medios de comunicación como forma de erradicar la discriminación y la violencia simbólica que estos ejercen cotidianamente. Asimismo, consideramos que la ampliación de sentidos inclusivos y de equidad a través de dichas normas podrían ratificar y acompañar muchas transformaciones socioculturales producidas en las últimas décadas en nuestro país. ${ }^{3}$

También hemos observado que, en los últimos años, la velocidad con la que ciertas retóricas igualitarias basadas en derechos han impregnado las agendas mediáticas y políticas se hizo evidente en cada nueva convocatoria protagonizada por el movimiento feminista desde el primer acontecimiento Ni Una Menos, el 3 de junio de 2015 a los Paros Internacionales de Mujeres, realizados los 8 de marzo de 2017 y 2018 (Camusso y Rovetto, 2016; 2017). Tras cada uno de estos acontecimientos masivos se ha podido complejizar el calado profundo de las desigualdades de género y sus manifestaciones concretas en todos los ámbitos, ubicando a los medios de comunicación y su racionalidad sexista en la mira.

En este recorrido hemos recuperado conceptualizaciones sobre la cultura profesional periodística como matriz que proporciona sentido y justifica las acciones emprendidas por la comunidad que labora en los medios de comunicación, planteando que quienes participan de esta cultura tienden a reproducir los valores dominantes de la sociedad a la que pertenecen en contenidos y prácticas internas (Melin-Higgins, 2004). Pero para algunas autoras feministas, el género como orden social, ${ }^{4}$ no sólo se reproduce en la cultura profesional, sino que además, es un componente estructural de la misma; es decir, impregna las prácticas, las relaciones, los contenidos y las personas que trabajan en los medios de comunicación. De ahí que se hable de gendered professional culture o cultura profesional generizada (Melin-Higgins, 2008).

Entonces, si el sentido del género opera mediante el orden simbólico de una dicotomía -masculinofemenino-, en la cual la pertenencia a alguna de las dos categorías marca diferencias en el ejercicio de poder (Blázquez Graf, 2010), podemos comprender de qué manera, en la cultura profesional de los medios de comunicación, se establecen clasificaciones sobre contenidos, perspectivas, habilidades, puestos, experiencias 
y valores que se normalizan a partir de una matriz dicotómica en el ejercicio de la profesión. El binomio masculino-femenino determina la asimetría social que la cultura profesional de los medios reproduce tanto en los contenidos como en las dinámicas internas de producción y que, al estar normalizadas por la propia cultura, se hacen difícil nombrar y, por tanto, modificar (Chaher y Santoro, 2007).

Desde estas perspectivas se puede calibrar el calado profundo de las desigualdades de género en las redacciones periodísticas y preguntarse por la persistencia de estas lógicas en los medios de comunicación alternativos, comunitarios, cooperativos o populares (en adelante, alternativos) ${ }^{5}$ que incorporan la participación de periodistas feministas y que pueden ser concebidos, a priori, menos lastrados por las asimetrías de poder hegemónicas. ${ }^{6}$

Si bien las teorizaciones sobre medios alternativos, contrahegemónicos, de la comunicación popular o autogestivos tienen varias décadas de desarrollo en nuestro país (Vinelli y Rodríguez Esperon, 2008; Segura, 2014; Segura y otrxs, 2018, entre otros), no se registran abordajes que problematicen las desigualdades de género en la organización laboral que los sostienen o que analicen la transversalidad de la perspectiva de género en sus prácticas y productos.

Para abordar la problemática planteada, proponemos un acercamiento exploratorio inicial a partir del desarrollo de seis entrevistas a mujeres periodistas que cuentan con experiencias de trabajo en este tipo de medios de producción local -radios, diarios, portales de noticias-. El criterio de selección procuró relevar testimonios de periodistas jóvenes, cuyas edades oscilan entre 25 y 35 años y que, además, forman parte de la colectiva Ni Una Menos de la ciudad de Rosario (en adelante, NUM), ${ }^{7}$ considerando que su acceso a la profesión coincide con el crecimiento de este tipo de medios y con la irrupción masiva de las demandas de los movimientos feministas y de la diversidad sexual.

Los contactos se establecieron en las asambleas convocadas para organizar el primer Paro Internacional de Mujeres en Rosario en las cuales participaban activamente. Las entrevistas fueron realizadas en encuentros de aproximadamente dos horas con una serie de preguntas abiertas que permitieron establecer una conversación distendida y el clima de confianza propicio para reconocer, a partir de las perspectivas singulares, las confluencias de sus experiencias de trabajo y militancia en tiempos de precarización laboral y reacción patriarcal.

De acuerdo con estos criterios, procuramos indagar sobre su participación profesional en la producción y organización de los espacios mediáticos alternativos. A su vez, teniendo en cuenta que la mayoría de ellas también cuenta con experiencia laboral en empresas mediáticas tradicionales, indagamos comparativamente las características percibidas en las rutinas de trabajo desarrolladas en ambos tipos de medios, atendiendo las diferencias de género en el reparto de tareas, roles y funciones, así como la inclusión de la agenda de género y diversidad en los contenidos informativos.

Antes de adentrarnos en el análisis de las entrevistas interesa, en primer lugar, ampliar una caracterización contextual, enfatizando la particularidad histórica de la constelación de factores que producen la emergencia actual de lo que se percibe como la "popularización de la discursividad feminista", en tanto reflejo de un movimiento plural y crítico; de ideas, denuncia y reivindicación, producto del conflicto social que genera una posición de subordinación y desigualdad de las mujeres y de otros sectores feminizados. Entendemos que estas discursividades emergentes vertebran sentidos y protagonizan acciones colectivas de denuncia y contestación a los límites que a su libertad establece la sociedad patriarcal, en una dinámica de profunda transformación cultural (de Titto, 2018; Montero, 2006).

En segundo lugar, analizamos los registros de las entrevistas realizadas, revisando las limitaciones y posibilidades que implica el trabajo periodístico en los medios alternativos desde la perspectiva de las propias trabajadoras, atendiendo la puesta en circulación de nuevas voces y temáticas emergentes, considerando las diferencias con los medios de comunicación tradicionales y los modos de organización del trabajo periodístico y su producto. 


\section{De LAS RETóricas igUALITARIAS AL PERIO-FEMINISMO}

Hemos apuntado más arriba que las desigualdades de género en los medios de comunicación se expresan en múltiples dimensiones a través de pautas culturales, criterios organizacionales, relaciones vinculares y prácticas cotidianas que dificultan el ejercicio profesional en igualdad de condiciones para varones y mujeres o personas con identidades feminizadas. A su vez, en los últimos años, se han puesto de manifiesto reclamos sectoriales por la nula implementación de los compromisos gubernamentales asumidos en declaraciones internacionales y normativas locales, orientados a garantizar el cumplimiento de estándares igualitarios en el ámbito laboral y en los productos resultante de la producción mediática. ${ }^{8}$

En estudios anteriores, a partir de datos relevados en encuestas realizadas a profesionales periodistas de la provincia de Santa Fe, ${ }^{9}$ hemos diagnosticado que la participación diferenciada de varones y mujeres en los escenarios laborales de los medios de comunicación es el resultado de la persistencia de una cultura periodística androcéntrica, incapaz de incorporar las transformaciones socioculturales y políticas producidas en las últimas décadas que incorporan las demandas y luchas históricas de movimientos sociales discriminados y marginalizados (Rovetto y Figueroa, 2015).

Ciertamente, transcurridos más de veinte años de la Declaración y la Plataforma de Acción de Beijing, la propuesta de igualdad de oportunidades entre varones y mujeres en los medios de comunicación, así como la posibilidad de hacerla efectiva en los ámbitos laborales no ha ido más allá de la retórica bienintencionada, coincidente con un periodo en el que aumentaron las condiciones precarias de contratación, reforzando desde el inicio de su inclusión formal en el mercado de trabajo, la discriminación sexual mediante mecanismos de segregación vertical y horizontal (Bárcena, 2010).

Este panorama se ha ido recrudeciendo con el impacto actual de las políticas de ajuste y achicamiento del sector mediático dejando como saldo espacios devastados para el desarrollo de las carreras profesionales cualificadas. ${ }^{10}$ Los efectos de este panorama, si bien impactan al conjunto de profesionales del campo periodístico -como en muchos otros sectores-, afectan diferencialmente a las mujeres (OIT, 2018). Es decir que, aun cuando en las redacciones, la presencia de las mujeres ha crecido cuantitativamente respecto a décadas pasadas, su entrada al sistema de empleo periodístico coincide con la precarización de las condiciones de trabajo, la amenaza constante del desempleo, la multiplicación de las formas de subcontratación parttime,freelancer, etc.- y, la consecuente, "rebaja" en materia de derechos laborales y salario (Henry, 2013; Rovetto, 2017).

No obstante, en el marco de este panorama desolador en el sector, en los últimos años el campo de estudios en el que centramos nuestras investigaciones, se vio conmovido por las prácticas y el activismo de perio-feministas. ${ }^{11}$ Identificamos allí una articulación política indispensable de carácter contra hegemónico y de disputa cultural frente a los discursos y prácticas androcéntricas dominantes, fundamentalmente, en los medios de comunicación masiva tradicionales. Esto implicó acercarnos, de forma analítica y también empática, a las historias de vida de comunicadoras jóvenes que participan de la escena mediática de la ciudad de Rosario, como productoras de contenidos informativos, al mismo tiempo que acompañan y forman parte de las luchas del movimiento social y político más dinámico del país en la actualidad.

Ciertamente, en este breve, pero intenso período hemos visualizado la emergencia y consolidación de cierto activismo político feminista en la profesión periodística que ha contribuido "desde adentro" de los medios tradicionales -y también desde otros medios y circuitos alternativos de comunicación e informacióna poner en la agenda pública temáticas cruciales vinculadas a los derechos de las mujeres y otros colectivos feminizados.

En la actualidad, contamos con múltiples ejemplos de esta creciente dinámica en el sector que nos permiten visualizar la cada vez más estrecha vinculación entre periodistas y movimiento de mujeres, así como reconocer 
los espacios y agenciamientos generados por estas trabajadoras, como es el caso de las periodistas nucleadas en colectivas NUM de distintas localidades del país.

Como síntoma de estas articulaciones, desde principios de 2018, en Argentina, los debates en torno a género, sexualidades y feminismos irrumpieron en programas de televisión abierta de la mano de reconocidas periodistas y comunicadoras feministas. La "penetración" del feminismo en la agenda mediática, su presencia en programas de televisión que miden alto rating, los hashtags \#AbortoLegalYa o \#SoyFeminista, como trending topic en twitter, en fin, la popularización de discursividades propias del programa de luchas feministas contribuyó con la amplificación de las discusiones sobre género y sexualidades que ya estaba en las calles, en las casas, las instituciones y todos los rincones (Alcaraz, 2018).

También, al compás de la organización de la segunda Huelga Internacional de Mujeres (\#8M), circularon en todo el arco mediático noticias, informes, notas de opinión que ponen en foco las reivindicaciones propias del sector. Aunque, en muchos casos, estas acciones fueron promovidas por las propias comunicadoras y periodistas, ya se venían manifestando con fuerza en medios informativos desde 2015 de la mano del accionar de NUM en distintas ciudades del país.

En el plano internacional y en el marco del \#8M, también se abrieron paso discursos y estrategias protagonizadas por trabajadoras del periodismo y la comunicación agrupadas en redes y asociaciones que permitieron instalar en la agenda internacional distintas dimensiones de la problemática. El uso de las redes y nuevas tecnologías -mediactivismo colaborativo-, globalizó múltiples iniciativas que fueron emergiendo en distintas partes del mundo para dar cuenta de la universalidad de las desigualdades de género en los escenarios periodísticos.

A nivel regional y local se destacan la articulación iberoamericana de la Red Internacional de Periodistas con Visión de Género (RIPVG), fundada en el año 2005, y Periodistas Argentina en Red (Red PAR), fundada en el año 2006. ${ }^{12}$

Al calor de las iniciativas generadas por la movilización del \#8M, se hicieron presentes distintas iniciativas promovidas por periodistas feministas y con visión de género que vale la pena reseñar. Entre ellas, el Manifiesto lanzado por un grupo de periodistas españolas, con el lema \#LasPeriodistasParamos que enumera las desigualdades que sufren las mujeres en el sector, y exhorta a las periodistas de todo el mundo a sumarse a la huelga general, de la siguiente manera:

Todas sufrimos el mismo machismo que las mujeres del resto de sectores - precariedad, inseguridad laboral, brecha salarial, techo de cristal, acoso sexual o ninguneos- pero con las particularidades asociadas a nuestra profesión. Somos conscientes de la relevancia social de nuestro trabajo y, por eso, mostramos también nuestra preocupación por la visión parcial de la realidad que tantas veces ofrecen los medios y en la que falta la presencia y aportaciones de las mujeres. El feminismo también es necesario para mejorar el periodismo (Las Periodistas Paramos, 2018).

Esta breve presentación y caracterización contextual nos permite situar el trabajo analítico que exponemos a continuación, donde procuramos recoger la perspectiva de las perio-feministas jóvenes integrante de la colectiva NUM Rosario para abordar aspectos vinculados con el tipo de medio en el que se desempeñan y la organización del trabajo al interior del mismo, las decisiones que toman en torno a la producción de contenidos, la articulación que desarrollan con redes de profesionales y sindicales, así como con su participación en espacios de militancia y activismo feministas y LGTBI.

Entendemos que reflexionar sobre este impulso llevado adelante, principalmente, por mujeres y personas con identidades de género no heteronormadas que trabajan en medios de comunicación no hegemónicos, se torna relevante tanto por el peso de los discursos informativos en sí, como por la interpelación al androcentrismo y la precarización laboral que estructura las relaciones y el trabajo en las organizaciones mediáticas tradicionales, como veremos más adelante.

Asimismo, tal como señala Segura (2018) retomando a Sel (2009), si los medios comunitarios, alternativos son proyectos comunicativos que se caracterizan por enfrentar tanto la agenda de los grandes conglomerados mediáticos y son reconocidos por diversos organismos internacionales ${ }^{13}$ que destacan su centralidad en las 
sociedades democráticas para garantizar el derecho a la comunicación, su estudio vinculado a la emergencia de los feminismos en la actualidad torna relevante este primer acercamiento exploratorio.

A pesar de no contar aún con estudios que hayan analizado los modos en que se despliegan las desigualdades de género en sus prácticas de producción, aquí podemos hipotetizar que dichos espacios se organizan a partir de lógicas de interacción laboral menos lastradas por relaciones de dominación basadas en la división sexual del trabajo. Y que, por ello, no reproducen punto por punto una mirada androcéntrica que cristalice el orden simbólico hegemónico, conservado y reforzado en las relaciones de poder que hemos identificado como parte de la cultura periodística desplegada en los espacios de producción más tradicionales (Rovetto, 2013).

\section{Perio-feministas abriendo espacios de comunicación alternativos}

Con lo expuesto hasta aquí, concluimos que las personas entrevistadas en la mayoría de los casos deben compatibilizar las experiencias de trabajo en medios alternativos ${ }^{14}$ con otros empleos en medios de comunicación hegemónicos o en áreas de prensa y comunicación de instituciones pblicas o privadas. Las periodistas entrevistadas también comparten características sociodemográficas comunes como ser solteras, vivir solas y sin familiares a cargo y han alcanzado titulaciones superiores en Periodismo y Comunicación Social. Todas ellas iniciaron su participación en medios de comunicación alternativos durante o ni bien concluyeron su formación.

En la mayoría de los casos, su participación en estos medios alternativos es casi ad honorem con escaso o nulo rédito en términos salariales. Este trabajo profesional no remunerado es visualizado como parte de la precarización general que afecta al sector, pero, al mismo tiempo es considerado, por algunas de las personas entrevistadas como un ejercicio "para foguearse" en la profesión y, fundamentalmente, como una práctica militante para todas las entrevistadas. Al respecto, una de las periodistas entrevistadas señala que la participación en estos espacios les posibilita producir contenidos desde la perspectiva de género con menos restricciones que en los medios tradicionales: "Es casi imposible poder sostenerlo, es difícil conseguir los recursos. La realidad es que la mayoría de nosotras trabajamos de otra cosa para vivir. Sin embargo, es allí donde mejores informes vinculados a las problemáticas de género he podido hacer" (Perio-feminista 1, comunicación personal, 15 de febrero de 2018).

Sin embargo, varias de las entrevistadas reconocen que su incorporación a este tipo de medios, no implicó per se la eliminación de prejuicios machistas a la hora de producir contenidos informativos, ya que aun en estos espacios alternativos se reproducen mecanismos discriminatorios en razón del género presentes en las rutinas laborales y la cultura periodística.

A su vez, estas experiencias se desarrollan en un contexto de crisis generalizada que atraviesa al sector en la ciudad de Rosario, como en el resto del país, y que va de la mano de la alta concentración mediáticoempresarial reforzada desde el inicio de este siglo (Smerling, 2010). Como consecuencia de este proceso, durante los últimos dos años, lxs trabajadorxs de prensa de todo el país han sufrido despidos, cierres de medios de comunicación y atraso en el pago de salarios. Según los sindicatos de prensa, la crisis que atraviesan los medios de comunicación en Argentina se ha agudizado desde la asunción de Mauricio Macri como presidente y se han perdido 2.500 puestos de trabajo en todo el país (FOPEA, 2017), aunque la crisis del sector no se inicia con este gobierno, se profundizó de forma creciente. Esto se evidencia en las transformaciones ocurridas en el mercado laboral en Argentina que se iniciaron en la década de 1990, con la llamada "flexibilización laboral" que agudizó el aumento de la desocupación, pero también, la subocupación y la precarización laboral de las nuevas generaciones que ingresaban al ámbito laboral de los medios de comunicación, con la incorporación de las pasantías, la colaboración externa, el trabajo free lance, entre otras formas desreguladas de empleo (Beccaria, 2005; Henry, 2013).

Con este panorama de fondo, las perio-feministas refieren que su ingreso profesional a medios autogestivos o cooperativos es, también, consecuencia de la crisis y concentración empresarial del sector que, si bien afecta 
al conjunto de lxs trabajadorxs, en sus casos particulares es percibida como un elemento de impacto diferencial en términos de colectivo generizado:

Cuando nos dimos cuenta que éramos todas mujeres, es porque somos las mujeres las que menos entran en los medios. Somos todas mujeres, todas profesionales, todas trabajan muy bien de lo que hacen, ya sea audiovisual o redactoras, y aun así hay muchas compañeras que no consiguieron ningún espacio donde puedan decir, yo laburo de periodista (Perio-feminista 5 , comunicación personal, 15 de febrero de 2018).

En la actualidad esta información coincide con la distancia que existe entre la cantidad de mujeres que año a año se gradúan en periodismo y comunicación social, y la cantidad de mujeres que efectivamente trabajan en empresas de medios (Rovetto y Figueroa, 2015). "Aunque sabemos que es excepcional, por el tipo de medio que somos, la mayoría de las redactoras y directoras somos mujeres (Perio-feminista 2, comunicación personal, 15 de febrero de 2018).

Como hemos visto hasta aquí, la situación laboral en estos espacios presenta signos de precariedad que refuerzan mecanismos de exclusión profesional que experimentan las mujeres en el conjunto de las organizaciones mediáticas. Esto es ilustrado por una de las entrevistadas cuando señala que los medios de comunicación alternativos se sostienen gracias al trabajo voluntario: "el (su) amor" a la profesión y el deseo de "poner a jugar sus conocimientos sin caer en un total abandono de sus perspectivas o, incluso, de las carreras profesionales" (Perio-feminista 5, ídem).

Asimismo, en sus testimonios se puede reconocer que la valoración positiva de los espacios de comunicación alternativos "para foguearse" en la profesión, así como los esfuerzos cotidianos que realizan para informar desde la perspectiva de género sin restricciones editoriales, colisiona con la ausencia de salario que garantice la subsistencia. A continuación, revisamos las percepciones de las periodistas entrevistadas en torno los cambios vividos en los últimos años en el sector y en sus prácticas, la persistente resistencia y dificultades con las que se enfrentan a diario y los vínculos y agenciamientos que logran construir a pesar de todo.

\section{4. "Para una Periodista no hay Nada MeJor que otra feminista"}

A pesar de todo, la experiencia colectiva y colaborativa entre redes y organizaciones de comunicadorxs y periodistas en las ltimas décadas demuestra la capacidad de desarrollar acciones conjuntas que favorezcan la inclusión de las reivindicaciones feministas en la agenda mediática. Sus prácticas innovadoras y creativas se valen de las posibilidades que brinda el contexto virtual para visualizar demandas, poner en circulación discursos contrahegemónicos, construir nuevos pactos y, en ese proceso, reinventar los modos del hacer y del decir frente a los modos de la desigualdad y opresión estructural derivadas orden patriarcal.

Sobre este aspecto, Segura (2014) ha señalado que los medios alternativos, desde su inicio, cuestionaron y reconfiguraron los roles de los sujetos de la comunicación: el productor o periodista profesional y los públicos, generando distintos niveles de radicalidad con pretensión de desintermediación y horizontalidad del proceso comunicacional. En este mismo sentido, la idea de democratizar la información —en su doble vía: producción y reconocimiento- ha tenido en las redes y el trabajo colaborativo el plafond para su materialización real:

Está bueno, hay que empezar a pensar estratégicamente al uso de redes, como periodistas, hay un montón de medios alternativos que son espacios de activismo, como Emergente o Midianinja, que hacen cosas increíbles gracias a las redes (Periofeminista 2, comunicación personal, 20 de febrero de 2018).

Esta perspectiva optimista sobre el lugar de las nuevas tecnologías y el uso de las redes sociales como nuevos espacios para comunicar y difundir que vienen a democratizar la circulación de información en la esfera pública, es estudiada por varias autoras desde hace algunas décadas (Laudano, 2016). 
A través del uso de redes sociales para difundir los contenidos que se producen en estos nuevos portales informativos, se ve una oportunidad para la puesta en circulación de nuevos sentidos sociales, entre ellos, la difusión de las ideas del feminismo.

Una de las entrevistadas, plantea el carácter estratégico de hacer uso de estas herramientas, propias del área de la publicidad y el marketing, para avanzar en un feminismo popular. "Explotar las herramientas que son propias de la publicidad y el marketing para usarlas en otro sentido. El feminismo necesita usar estas técnicas para ser cada vez más masivo" (Perio-feminista 6, comunicación personal, 15 de febrero de 2018).

Estas intervenciones pueden ser pensadas desde una perspectiva diferente a partir de lo sucedido durante el 2018 y la irrupción del feminismo en la agenda mediática. La masividad en las nuevas convocatorias a asambleas de mujeres de cara a la organización del \#8M del 2019 dan cuenta del avance que implicó para el feminismo ponerse a dialogar con sectores de la sociedad que hasta ahora habían estado fuera de su alcance.

Al preguntar sobre la llegada masiva que tienen las redes sociales y el impacto que pueden llegar a tener los portales de noticias feministas, una de las entrevistadas nos comparte su visión pesimista sobre las limitaciones de poner a circular contenidos sin conocer su verdadero alcance.

Con el tema de la red social lo que pasa es que, no sé quién lee lo que se comparte, yo no sé hasta qué punto no se generan como estos microcircuitos donde uno piensa que hay un montón de gente de acuerdo con un montón de cosas. Porque son todos sus amigos (...) Es súper valioso que las feministas usemos las redes, pero cabe preguntarnos por qué llegada tienen realmente, si queremos tener algún tipo de incidencia masiva (Perio-feminista 3, comunicación personal, 30 de febrero de 2018).

Al mismo tiempo, otra entrevistada nos comparte que la existencia de portales feministas sirve al trabajo de periodistas que lo usan como fuentes en sus notas, pero desconfía de que esos espacios alcancen para popularizar las discursividades feministas.

Yo trabajo diariamente con portales de noticias feministas, lo uso como fuentes, para estar informada. En mi caso, también destaco la solidaridad que existe entre las mujeres que trabajamos en este sector. Para una periodista comprometida no hay nada mejor que otras periodistas comprometidas. Sin embargo, también creo que la diferencia con otros espacios es que nos leemos entre nosotros. Funciona como una especie de lugar de confort, de circuito cerrado (Perio-feminista 4, comunicación personal, 20 de febrero de 2018).

Por otra parte, el reflexionar sobre la cultura organizacional de este tipo de medios habilita a revisar los modos de circulación de poder y su vínculo con las relaciones de género. Este aspecto es referenciado por algunas entrevistadas en relación con los avances del movimiento feminista en la actualidad, de donde se nutren para interpelar las desigualdades en los lugares de trabajo. "En los últimos años, se fueron dando procesos de empoderamiento de las mujeres en estos espacios y eso nos ha dado un mayor entrenamiento para ocupar lugares que en otros medios más tradicionales sería imposible" (Perio-feminista 3, comunicación personal, 20 de febrero de 2018).

$\mathrm{Al}$ mismo tiempo, se reconocen las limitaciones de conformar medios que no son hegemónicos, pero intentan hacer sonar otras campanas, poner en circulación contenidos informativos en estos medios emergentes. "En el contexto actual, hemos logrado tener una voz más fuerte. Y ahora por lo menos en las cooperativas, las mujeres tienen otro lugar, se ganaron ese respeto" (Perio-feminista 1, ídem). Es que en estos espacios también se colaron las discusiones sobre feminismos, espacios donde se intentaejercer otro modo de hacer periodismo, tuvieron que incluir de una manera diferente la voz de las mujeres.

A pesar de que muchas veces el número de mujeres al que se alcanza con este tipo de contenidos sea reducido, los medios alternativos han sido, históricamente, una especie de trinchera para las feministas, espacios desde los cuales se ponen en marcha la circulación de discursos disidentes sobre sexualidades.

Aquí consideramos que esta falsa dicotomía entre "mantener la autonomía", produciendo contenidos desde la autogestión (que implica siempre también una suerte de explotación -jotra!-) vs. filtrarse en la grieta en los medios masivos (y colar contenidos feministas en los medios tradicionales), si bien sirve para preguntarse sobre las dinámicas del movimiento en la actualidad, también permite ponderar las diversas 
estrategias de acción y herramientas que se construyen para potenciar la perspectiva de género en los contenidos informativos.

Según las perio-feministas, los aportes a la circulación de nuevos sentidos y significaciones en relación a sexualidades, roles de género, pueden y deben venir desde cualquier lugar, tengan el alcance que tengan; mientras que aparezcan voces en disidencia, estaremos hablando de contrainformación. "No importa si lo hacemos desde Twitter, desde un medio tradicional o desde un medio alternativo,cualquier aporte que sirva para poner en agenda temáticas y discursos feministas, es válido en este momentopara el movimiento de mujeres" (Perio-feminista 4, idem). Esta perspectiva indica que la puesta en circulaciónde discursividades feministas en la agenda pública implica per se un avance para este movimiento. Implicala posibilidad de habilitar el diálogo, de nombrar las problemáticas que atraviesan las mujeres visibilizar lasdiscriminaciones, desde una trayectoria de profundice yendo incluso más allá de visibilizar las violencias sexistas.

Nos capacitamos en el diario cuando nos hicimos cooperativa y nos propusimos escribir desde la perspectiva de género, no solo las notas que hablen sobre mujeres sino cualquier tema... lo cual te da un dolor de cabeza grande. Yo siento que ser feminista en el diario implica más trabajo y estar siempre en batalla, si o sí, porque siempre tus compañeros o directorxs te evalúan más (Perio-feminista 4, idem).

El recorrido de una de las entrevistadas, según nos indica, la llevó a dejar de pensar la violencia de género como una temática aislada, y empezar a preguntarse por la implicancia de las violencias sexistas en la totalidad de las relaciones sociales y la necesidad de atravesar cualquier contenido informativo con perspectiva de género y con las perspectivas situadas de las personas que los producen. "Mi búsqueda, ahora, tiene que ver con cómo atravesar toda la agenda. Desde los baches en las calles, la vivienda o la economía con una perspectiva de género" (Perio-feminista 2, idem).

Desde el principio, asumimos que somos un medio feminista porque somos conscientes de lo que está pasando en el mundo en el que vivimos, porque las reivindicaciones históricas hoy están en agenda más que nunca, pero también porque queremos ser identificadas como feministas y no creemos en el periodismo neutral (Perio-feminista 6, comunicación personal, 15 de febrero de 2018).

Aun así, y a pesar de reconocer todos estos avances en materia de elaboración de contenidos y posicionamiento respecto de los sentidos que los mismos evocan, reconocen que la información de "género" aparece como aislada, desprendida del material significante a trabajar por el conjunto de lxs profesionales. En los casos que comparten el ejercicio profesional con varones, las entrevistadas reniegan de ser ubicadas en el lugar de "especialistas" sobre "género", mientras que los varones se desentienden de elaborar dichos contenidos. "Por un lado, parece que a las cuestiones de género las tienen que tratar sólo las mujeres y, por otro, que les dicen:como son ustedes las que hinchan con esto, cubran solas esas noticias" (Perio-feminista 3 , ídem).

Hasta aquí con este acercamiento exploratorio hemos podido constatar que el trabajo profesional que cotidianamente emprenden estas jóvenes periodistas, así como su participación activa en el movimiento de mujeres va de la mano de la articulación y la conformación de espacios de encuentro, contención y organización entre las mujeres con quienes comparten perspectivas y estrategias. Tejer redes de intercambio, establecer nuevos pactos sectoriales basados en vínculos solidarios con otras colegas mujeres que están atravesando situaciones similares, es parte de las estrategias de supervivencia cotidianas que estas periofeministas llevan adelante en un contexto cada vez más adverso para el ejercicio profesional.

\section{CONSIDERACIONES FINALES}

Este primer acercamiento al vínculo entre feminismos y medios alternativos, centrándonos en las experiencias de las mujeres jóvenes periodistas de la ciudad de Rosario nos permitió reconocer aspectos del fenómeno estudiado, habilitando renovar interrogantes y reflexiones sobre la profesión periodística y los 
escenarios de producción de contenidos socialmente relevantes. Ahondar en el papel del Estado nacional y provincial para el fortalecimiento o no de las experiencias de comunicación alternativas, la vinculación con las organizaciones políticas y sindicales -en un contexto de ajuste sin precedentes que afecta al sector de la comunicación y el periodismo-, así como indagar sobre el rol de los varones en estos procesos, constituyen, sin dudas, claves de análisis para futuras inmersiones en este particular campo de indagación.

Del análisis de las entrevistas a las perio-feministas integrantes de NUM Rosario, se puede desprender que, a pesar de la adversidad con las que se encuentran a diario, tanto por los condicionantes externos como por las desigualdades con las que conviven en los propios ambientes de trabajo, estas jóvenes profesionales se han constituido como referentes de la lucha del movimiento de mujeres a nivel local realizando prácticas profesionales de carácter contrahegemónica. Dichas prácticas reflejan las alianzas construidas en los últimos años entre las organizaciones de mujeres y activistas con las periodistas del campo que se materializan en agenciamientos políticos y en la producción misma de contenidos informativos.

De ahí que la expresión de "minoría bulliciosa" -además de ser una paráfrasis que invierte el sentido de la noción sociopolítica "mayoría silenciosa”, referida al conjunto de la población que no expresa su opinión en público" - procure retratar un colectivo de mujeres jóvenes que se abre camino en contextos de alta precarización, sosteniendo espacios mediáticos de alcance limitado y escasa rentabilidad, frente a los grandes medios tradicionales en la ciudad.

En sus experiencias vitales también se conjugan esfuerzos cotidianos por compatibilizar el activismo político con el desarrollo profesional. Los costos profesionales y subjetivos que implica conciliar estos aspectos se traducen en mayor desgaste y precariedad, en la falta de reconocimiento por las tareas realizadas y en la necesidad de estar, permanentemente, a la defensiva para sostener desde una práctica profesional con perspectiva de género.

Consideramos que este particular oficio implica hoy reconocimientos de carácter social y cultural vinculados a la relevancia adjudicada a la comunicación como campo de intervención cultural y política, como usina de producción y de disputas constantes de sentidos que se ubican en el centro de los debates públicos. Es por esto que interesa continuar revisando las formas actuales de ejercicio profesional, identificando su incidencia en el contexto sociocultural más amplio que reclama sentidos inclusivos, democráticos y plurales.

\section{REFERENCIAS}

Alcaraz, M. F. (2018). El rating es feminista. Post en Anfibia. Recuperado de: http://www.revistaanfibia.com/ensay o/el-rating-es-feminista

Amado, A. (2012). Periodismo argentino: en busca de datos sobre la profesión. Austral Comunicación, 1(2), 113-135. Recuperado de: http://www.austral.edu.ar/ojs/index.php/australcomunicacion/article/view/27

Amado, A.; Bongiovanni, M. y Dragonetti, M. (2016). Periodismos argentinos: perfiles y contextos. En Amado Suárez, A., et al., Periodismos argentinos: modelos y tensiones del siglo XXI (pp. 123-153). Buenos Aires: Konrad Adenauer Stiftung.

Bárcena, A. (coord.) (2010). ¿Qué Estado para qué igualdad? Documento para la XI Conferencia Regional sobre la Mujer de América Latina y El Caribe. Santiago de Chile, Chile.

Beccaria, L. (2005). Jóvenes y empleo en la Argentina. En Anales de la educación común, 1(1,2), 177-182.

Blázquez Graf, N. (2010). Investigación feminista. Epistemología metodológica y representaciones sociales. México D. F: Universidad Nacional Autónoma de México.

Camusso, M.y Rovetto, F. (2016). \#Ni una (imagen) menos. Imágenes, apropiaciones y circulación en las redes sociales. En Cingolani, G. (Ed.). Nuevas mediatizaciones: nuevos públicos: cambios en las prácticas sociales a partir de las transformaciones del arte y los medios en la red (pp. 159-177). Rosario: UNR Editora. 
Camusso, M. y Rovetto, F. (2017). Naturaleza y artificio: Iconografías de mujeres políticas en la bisagra de un "cambio de ciclo”. En Camusso, M. y Busso, M. (Eds.). Mediatizaciones en tensión. El atravesamiento de lo público (pp. 86 - 106). Rosario: UNR Editora.

CEPA (2018). Centro de Economía Política Argentina. Recuperado de: http://centrocepa.com.ar/informes.html Chaher, S. y Santoro, S. (2007). Las palabras tienen sexo II. Buenos Aires: Edit. Artemisa Comunicación.

Chaher, S. (2014). Politicas públicas de comunicación y género en América Latina: un camino por recorrer. Buenos Aires: Comunicación para la Igualdad Ediciones; Friedrich Ebert Stiftung.

de Titto, J. (2018). Crear un nuevo mundo también es narrarlo. En Freire, V (comp.) La cuarta ola feminista (pp. 55-64). Buenos Aires: Oleada / Malajunta.

FOPEA (2017). Informe Especial 2016 del Observatorio y alerta laboral de periodistas. En Michi, G. y Berghella, V. (coord.). Crisis en los medios y situación laboral de los periodistas en 2016 (pp. 8-17). Buenos Aires: FOPEA.

Gallego, J. (comp.). (2002). La prensa por dentro. Producción informativa y transmisión de estereotipos de género. Barcelona: Libros de la Frontera.

Henry, L. (2013). Fragmentación y precarización laboral en la prensa escrita. Los desafíos para la representación y la organización colectiva de los periodistas en un entorno productivo flexibilizado. En Senén González, C. (comp.). La revitalización sindical en Argentina: alcances y perspectivas (pp. 201-214). Buenos Aires: Prometeo.

Las periodistas paramos (2018). Manifiesto. Trabajadoras del periodismo y la comunicación nos sumamos a la huelga del 8M. Recuperado de https://lasperiodistasparamos.wordpress.com

Laudano, C. N. (2016). Feministas en 'la red'. Reflexiones en torno a las potencialidades y restricciones de la participación en el ciberespacio. En Rovetto, F. y Fabbri, L. (ed.) Sin feminismos no hay democracia (pp. 31-54). Rosario: Último Recurso.

López Diez, P. (2004). La mujer, las mujeres y el sujeto del feminismo en los medios de comunicación. En López Diez, P. (ed.). Manual de información en género (pp. 107-143). Madrid: IORTV (RTVE) e Instituto de la Mujer.

Martini, S. y Luchessi, L. (2004). Los que hacen la noticia. Buenos Aires: Biblos.

Melin-Higgins, M. (2004). Coping with journalism: Gendered newsroom culture in Britain. In de Bruin, M. and Ross, K. (Eds.). Gender and newsroom cultures: Identities at work (pp. 197-222). New York: Hampton Press.

Melin-Higgins, M. (2008). Gendered journalism. Cultures strategies and tactics in the fields of journalism in Britain and Sweden. Malmö: Department of Journalism and Mass Communication

Mellado, C; Moreira, S; Lagos, C; Hernández, M.E. (2012). Comparing journalism cultures in Latin America: the case of Chile, Brazil and Mexico. International communication gazette, 74, 60-77.

Montero, J. (2006). Feminismo: un movimiento crítico. Intervención psicosocial, 15 (2), 167-180.

ONU, OEA, OSCE y CADHP (2007). Declaración conjunta sobre diversidad en la radiodifusión. Recuperado de ht tp://www.osce.org/es/fom/29826

OIT (2018). Perspectivas sociales y del empleo en el mundo: avance global sobre las tendencias del empleo femenino 2018. Ginebra: Oficina Internacional del Trabajo.

Ortega, F. y Humanes, M. L. (2000). Algo más que periodistas. Sociología de una profesión. Barcelona: Ariel.

Papí Gálvez, N. (2008). El género entre bastidores. El caso de las periodistas de la Comunidad Valenciana. Alicante: Universidad de Alicante.

Postolski, G; Santucho, A. y Rodríguez, D. (2002). Concentración y dependencia: los medios de comunicación en el centro de la crisis. Buenos Aires: Observatorio Político y Social de Medios y del Área de Investigación de la Unión de Trabajadores de Prensa de Buenos Aires.

Rosenberg, L. (2015). Los inicios de la carrera periodística: entre la formación académica y la formación en la práctica. Questión. 1 (46), 431-440.

Rosenberg, L. (2016). Sociabilidad y mundo del trabajo: El caso de la sala de redacción de un diario. Trabajo y sociedad. $27,365-385$.

Rovetto, F. (2013). Percepciones sobre desigualdades de género en el trabajo periodístico. Globalmedia journal México; $10(20), 54-73$. 
Rovetto, F. (2017). "Igualdad de oportunidades y trabajo periodístico". Retos, retóricas y obstáculos: revista internacional de comunicación y desarrollo, 7(2), 35-46.

Rovetto, F. y Figueroa, L. (2015). La cocina del trabajo periodístico. Desigualdades de género y distribución de poder en las redacciones informativas de la provincia de Santa Fe. Question. 1(48), 494 - 509.

Rovetto, F. y Figueroa, L. (2018). Perio-feminismo desde adentro. Desigualdades de género en los medios. Con X, 4. DOI: https://doi.org/10.24215/24690333e022

Smerling, T. (2010). Diez años de concentración de la propiedad de los medios en Rosario Del decano de la prensa argentina al multimedio La Capital. Signo y pensamiento. 29(57), 520-536.

Scott, J. (1996). El género: una categoría útil para el análisis histórico. En Lamas, M. (comp.) El género: la construcción cultural de la diferencia sexual (pp. 265-302). México: PUEG.

Segura, M. S. (2014). Desintermediación y participación. Los nuevos roles de periodistas y públicos en los medios no lucrativos. La trama de la comunicación, 18, 115-133.

Segura, M. S. y otrxs. (2018). La multiplicación de los medios comunitarios, populares y alternativos en Argentina. Explicaciones, alcances y limitaciones. Revista internacional de comunicación y desarrollo, 9, 88-114.

Sel, S. (2009). Comunicación alternativa y políticas públicas en el combate latinoamericano. En Sel, S. (coord.). La comunicación mediatizada: hegemonias, alternatividades, soberanias (pp.13-36). Buenos Aires: CLACSO.

Si.Pre.Ba. (2018). Sindicato de Prensa de Buenos Aires. Recuperado de: http://www.sipreba.org/

Soriano, J. (2005). La incorporación de la mujer a la profesión periodística en España: crítica a la hipótesis de la feminización.Revista F@ro, 2, 353-368.

Vinelli, N. y Rodríguez Esperón, C. (comp.) (2008). Medios alternativos para la acción política. Buenos Aires: Continente.

WACC (2015). Proyecto de Monitoreo Global de Medios. Ottawa: UNIFEM.

\section{Notas}

1 También se pueden mencionar otros estudios relevantes en el país y la región que, si bien no centran su mirada en las desigualdades de género en el sector, aportan datos cuantitativos y reflexiones teóricas para comprender los cambios en las dinámicas laborales y la cultura profesional de las organizaciones informativas (Amado, 2012; Martini y Luchessi, 2004; Mellado, Moreira, Lagos y Hernández, 2012; Rosenberg, 2015; 2016, entre otrxs).

2 Ambas leyes fueron sancionadas en el año 2009. La primera de ellas colocó a nuestro país a la par de otros de la región que ya habían comenzado a sancionar leyes de violencia de "segunda generación", más acordes al marco propuesto por la Convención Interamericana para Prevenir, Sancionar y Erradicar la Violencia contra la Mujeres (Belém do Pará). La segunda, procuró valorización del derecho a la comunicación, mediante la redistribución del poder, de los recursos económicos y, también, de los recursos simbólicos. De la confluencia de ambas normativas se fraguó un acuerdo entre distintos organismos del Estado para declarar el Año de Lucha contra la Violencia Mediática hacia las Mujeres y la Discriminación de Género en los Medios Audiovisuales en 2014 (Chaher, 2014).

3 La Ley de Servicios de Comunicación Audiovisual fue modificada por el Decreto de Necesidad y Urgencia (DNU) $267 / 2015$, firmado en diciembre de 2015, por el actual jefe del ejecutivo nacional, Mauricio Macri. Esta modificación ha implicado un retroceso en materia de derechos, interrumpiendo su efectiva aplicación, dejando sin efecto el desarrollo de medidas que puedan favorecer, entre otras cosas, la inclusión de la perspectiva de género en los medios de comunicación. Al mismo tiempo, el mencionado DNU, disolvió el Consejo Federal de Comunicación Audiovisual y el Consejo Federal de Tecnologías de las Telecomunicaciones, que incorporan la activa participación de representantes de los sindicatos, las universidades y las provincias (Rovetto y Figueroa, 2018).

4 Se recupera aquí la acepción del género como un orden simbólico constitutivo de las relaciones sociales (que son relaciones de poder) basadas en las diferencias que distinguen los sexos (Scott, 1996).

5 No existe un consenso cerrado para nombrar a este tipo de medios que pueden ser nombrados como alternativos, comunitarios, populares, de gestión cooperativa, etc. En esta comunicación, definimos a las experiencias analizadas como medios "alternativos" destacando su impronta diferencial de los medios masivos tradicionales.

6 La historia de los medios "alternativos" de comunicación cuenta con una larga y variada experiencia en nuestro país, creciendo a través de redes y organizaciones sociales siempre en los márgenes de la industria de la comunicación hegemónica. En los últimos años, las emisoras radiales y señales de televisión contaron con reconocimiento y políticas de 
promoción a partir de la sanción de la Ley n 26.522 en 2009 (Segura et al., 2018). Por su parte, los portales de noticias y periódicos online gestionados de manera autónoma o cooperativa proliferaron a partir del desarrollo de las nuevas tecnologías de acceso masivo. Esto se ha hecho evidente en los últimos años, con la creación de listas de distribución y portales informativos a cargo de jóvenes comunicadores y periodistas (Laudano, 2016).

7 Respecto al colectivo NUM que, por su impacto social y configuraciones particulares en distintas ciudades del país, merecería una caracterización más detallada, diremos que, en el marco de su etapa de emergencia pública, convocando a la movilización del 3 de junio de 2015 con la consigna \#BastaDeFemicidios, se organiza a partir de la conformación de un grupo de periodistas y comunicadoras reconocidas por su trabajo en medios prensa. En la página oficial de NUM Capital Federal se presentan las integrantes que conformaron el colectivo en sus inicios. Actualmente, las distintas configuraciones locales, incorporan integrantes de diversas procedencias. En la ciudad de Rosario, la colectiva NUM presenta una configuración intergeneracional, está integrada por periodistas, artistas, investigadoras, docentes y activistas. Más información disponible en: https://www.facebook.com/niunamenosenrosario/ .

8 Nos referimos a declaraciones como las emanadas de la Conferencia de Beijing (1995), así como las Conferencias Mundiales sobre la Mujer $(1975,1980,1985,1995)$, recogidas a partir de la década del '90 en nuestra región donde abundan documentos con posturas ejemplares a favor del principio de igualdad de oportunidades para ampliar los derechos de las mujeres en todos los órdenes de la vida. Como ejemplo podemos mencionar que, en el año 1998, el MERCOSUR crea la Reunión Especializada de la Mujer (REM), postulando "la conveniencia de establecer un ámbito de análisis de la situación de la mujer respecto a la legislación vigente en los Estados Partes, en lo que se refiere al concepto de igualdad de oportunidades". También a nivel local, el Estado argentino aprueba el Plan para la Igualdad de oportunidades entre Varones y Mujeres en el Mundo Laboral en el año 1998, mediante Decreto nacional No 254/98. Estas declaraciones y principios formaron parte del espíritu con el que se confeccionó la Ley de Servicios de Comunicación Audiovisual (Ley 26.255) aprobada en 2009 y modificada por el Decreto en 2015, firmado por el actual jefe del ejecutivo nacional, Mauricio Macri.

$9 \mathrm{Al}$ circunscribir el estudio en la provincia de Santa Fe tuvimos en cuenta también la implicancia del marco normativo particular impulsado desde el Estado provincial a partir de la aprobación del Plan Estratégico de Igualdad de Oportunidades y Derechos (2013-2020) que incluye desarrollar políticas de equidad en los medios informativos y otras organizaciones que componen el mapa de producción de la industria cultural a nivel local.

10 Sobre el impacto de las políticas de ajuste económico a nivel nacional implementadas por el actual gobierno nacional y sus efectos en los medios de comunicación tanto públicos como privados hay una vasta cantidad de información disponible con datos estadísticos y análisis coyunturales, entre los cuales podemos destacar los informes elaborados por el Centro de Economía Política (CEPA, 2018), y los informes elaborados por el Sindicato de Prensa de Buenos Aires (SiPreBA, 2018).

11 Con el término perio-feministas nos referimos a mujeres que se autodefinen como periodistas y feministas, y que hacen de sus prácticas infocomunicacionales un ejercicio profesional y militante al mismo tiempo (Rovetto y Figueroa, 2018).

12 Como antecedentes de estos espacios de articulación podemos ubicar a las agencias de noticias feministas y con enfoque de género surgidas en la década del ' 90 . Entre ellas se destaca, por su alcance y duración, la mexicana CIMAC (Comunicación e Información en la Mujer), que fue la primera agencia informativa especializada en difundir una agenda regular con perspectiva de género y, más adelante, la española AMECO PRESS (de la Asociación Española de Mujeres Profesionales de los Medios de Comunicación). Ambas agencias se articulan con otros espacios similares de reciente creación y sus contenidos informativos tienen proyección internacional.

$13 \mathrm{Al}$ respecto, Segura (2018) enfatiza los estándares internacionales proveídos por organismos como la ONU, OEA, OSCE y CADHP (2007), que han identificado este tipo de medios como indispensables el fortalecimiento del tejido social, la representación de diferentes identidades culturales y de construcción de la democracia, sostienen que las condiciones de posibilidad del sostenimiento de los medios comunitarios son una cuestión pública que el Estado debe garantizar.

14 Los medios de comunicación alternativos en los que trabajan son, principalmente, portales de noticias online, aunque alguno de ellos también se distribuye en formato papel. Estos son: Redacción Rosario y El Eslabón de Cooperativa de Trabajo La Masa, que funciona desde el año 2010; diario El ciudadano y la Región que en el año 2016 deja de pertenecer al Grupo Indalo para convertirse con cooperativa gestionada por sus trabajadores; Agencia Sin Cerco, que se presentan como "un medio de comunicación autogestivo, conformado a inicios de 2016 por militantes de distintos espacios sociales, políticos y gremiales: periodistas, fotógrafxs, comunicadorxs y realizadorxs audiovisuales”. Todos ellos iniciaron su actividad gracias a distintas iniciativas colectivas. Si bien no nos detendremos aquí en la descripción de sus características particulares, diremos que presentan grandes variaciones en cuanto a la composición y organización interna del trabajo, pero pueden ser pensados como una unidad en tanto no forman parte de grupos empresariales o multimedios privados que, en términos económicos, se caracterizan por la concentración como estrategia de expansión en la búsqueda por dominar el sector (Postolski, Santucho y Rodríguez, 2002). 